\title{
Using behavioural change as a tool to reduce healthcare associated infections in a low resource setting
}

\author{
Namita Jaggi, Pushpa Sissodia \\ Artemis Health Institute, Sector 51, Gurgaon, Haryana-122001, India
}

doi: 10.3396/IJIC.v10i4.027.14

\begin{abstract}
Infection Prevention is an old science but a new art. The reasons for non-compliance to infection control practices in healthcare settings are related to psychological barriers, preconceived notions, cultural influences and ineffective time and resource management. Can we use behavioural change as a powerful tool to improve infection control practices and reduce healthcare associated infections (HAI)? This study was carried out for one year from July 2012 to June 2013. Twenty four healthcare practitioners (nurses) were identified. A baseline assessment of their awareness and compliance in infection prevention was undertaken followed by a behavioural training imparted by a psychologist who then addressed the issues. A post training assessment was conducted and results statistically analyzed. Results showed that 19 of the 24 (79\%) showed significant improvement $(\mathrm{p}<0.05)$ in all aspects of competency in infection prevention. A positive correlation was observed between the compliance to infection control practices and decrease in HAI. In conclusion, understanding human behaviour leading to low compliance to infection control practices and imparting training to overcome these blocks can be a powerful, cost effective and a rational approach to reduce $\mathrm{HAl}$ in low resource settings.
\end{abstract}

Keywords: Patient care team and education; Quality improvement; Cross infection and prevention and control; Attitude of health personnel

\section{Corresponding Author}

Dr. Namita Jaggi

Director, Labs \& Infection Control

Artemis Health Institute, Sector 51, Gurgaon, Haryana-122001, India

Email: namita@artemishealthsciences.com 


\section{Introduction}

Health-care associated infections (HAls) and increasing antimicrobial resistance (AMR) are growing global concerns which impact patient care, patient safety and ultimately the quality of care. ${ }^{1}$ Development of an effective infection prevention and control (IPC) program with a focus on standardized protocols and continuing staff education does help in the reduction of these infections. ${ }^{2}$ However, a review of evidence has concluded that well documented guidelines and policies have not always translated into best practices. ${ }^{3-5}$ Compliance to IPC precautions and hand hygiene is internationally suboptimal. ${ }^{6}$ Even though the healthcare workers know and understand the institutional policies, social, cultural and environmental factors affect their behaviour which in turn affects the translation of these policies into practice. ${ }^{7}$

This has so far not been effectively addressed and it is important that interventions which bring about changes in behaviour are adopted and utilized in healthcare settings. A wide range of behaviour change models have been developed and adapted for use in programs which have involved studying the components of the behaviour system that are required to bring about an effective change. ${ }^{8}$

To assess, formulate and apply behavioural change as a tool to reduce $\mathrm{HAI}$ in a low resource setting, we designed a one year study in an Indian tertiary intensive care unit (ICU). The main objectives of the study were to evaluate the perceptions, attitude and mindset of nurses, to identify the important barriers preventing adoption of IPC practices, to impart specific behavioural training focusing on removing those barriers and ultimately creating a culture of safety within the organization. The post training improvements in hand hygiene and IPC protocols compliance were measured and correlated with the HAI rates.

\section{Methods}

\section{Setting}

This cross-sectional study was undertaken between July 2012 and June 2013 in 4 ICUs [MSICU1 (Medical Surgical ICU)-36 Beds, MSICU2-20 Beds, PICU (Paediatric ICU)-12 beds and NICU (Neonatal ICU)-10 beds] of a tertiary care hospital setting in India.

\section{Design:}

The study was divided into the following stages (Fig 1):

1. Stage I (July-Sep 2012)- Selection of participants: 47 nurses with more than 5 years of experience were randomly selected by draw of lots by ICU Head and were subjected to answer a questionnaire which covered questions related to knowledge about infection control measures namely hand hygiene and prevention of specific device associated infections (catheter associated urinary tract infection [CAUTI], central line associated blood stream infections [CLABSI] and ventilator associated pneumonia [VAP]). There were 20 questions carrying 2 marks each. The nurses were selected on the ability to score at least $50 \%$ marks. There were 24 such nurses selected.

2. Stage II (October-December 2012)- IPC practice measurement: All the selected (24) nurses were then observed for adherence to hand hygiene and compliance to an infection control (IC) check list for device associated infections. The hand hygiene compliance was measured by the infection control nurses (ICN) as per the $\mathrm{WHO}^{9}$ recommended method. These ICNs were trained suitably about the Infection control practices by the Infection Control Officer (ICO) followed by a competency test. The Hand hygiene and checklist compliance collected by ICNs was suitably corroborated, checked and validated by the ICO. The IC checklist focused on 5 critical selected parameters for prevention of each of the specific device associated infections with 1 point awarded for compliance to each critical component. The hand hygiene and IC checklist compliance percentages of the respondents were then averaged (mean) and recorded.

The percentage compliance was calculated in both these sections as follows:

Hand Hygiene Compliance (\%):

Total no. of Hand Hygiene actions x 100

Total no. of Hand Hygiene opportunities

\section{IC Checklist Compliance (\%):}

No. of IC measures adhered to $\times 100$

Total no. of IC measures recommended 
3. Stage III (January-March 2013)- Behavioural assessment and Training: The selected candidates were subsequently subjected to a Behavioural Assessment Scale (BAS) where a 5-point Likert scale $(1=$ Strongly agree, $2=$ Agree, $3=$ neither agree nor disagree, $4=$ disagree and $5=$ Strongly disagree) was applied on 25 questions to analyze the perceptions, attitude and mindset of nurses

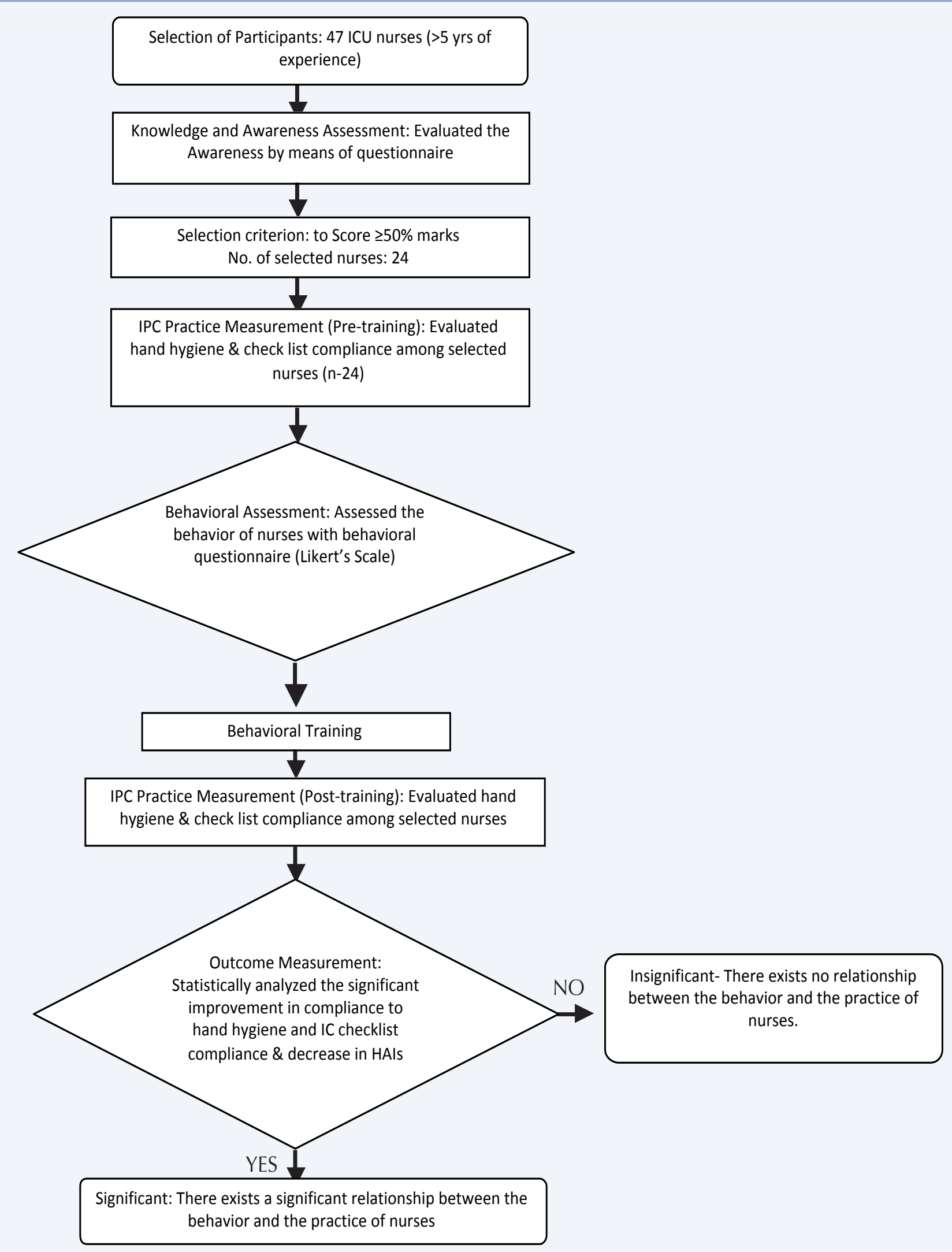

Figure 1. Process Flow of the study 
towards IPC. The 5 most common barriers were listed by picking up what most respondents ticked as agree or strongly agree. Post behavioural assessment, specific training targeted to overcome the 5 identified stumbling blocks was provided by a trained psychologist.

4. Stage IV (April-June 2013)- IPC practice measurement: The effect of behavioural training in removal of the stumbling blocks was assessed by subjecting the 24 nurses to observation of compliance to hand hygiene and IC checklist again.

5. Outcome measurement: The pre- and post-training hand hygiene and IC checklist compliance and the HAls were compared, analyzed and correlated for the entire study period. The HAI surveillance protocols and guidelines for all the four infections like CAUTI, CLABSI, VAP and surgical site infection (SSI) were based on the $\mathrm{CDC}$ recommendations. $\mathrm{HAI}$ surveillance was performed by the ICNs as part of their routine work which involved collection, analysis and reporting of data on healthcare associated infections. The suspected HAls were then confirmed and validated after discussion with the treating clinician and the ICO.
6. Data analysis: Statistical analysis was performed by SPSS-20.0 software. A paired sample t-test was performed on the compliance to hand hygiene and IC checklist and HAI rates for pre- and postbehavioural training periods. A p-value of $<0.05$ [at $95 \%$ confidence interval] was considered significant.

\section{Results}

A total of 24 out of $47(51 \%)$ nurses with an experience of 5 years or more in the ICU were selected for the study by means of a baseline assessment of their knowledge and awareness of hand hygiene and specific device associated infections.

\section{IPC practice measurement-Pre training}

During the IPC measurement in the pre-training period, the mean hand hygiene compliance of the 24 nurses ranged from $30 \%$ to $55 \%$, the mean being $40 \%$. The IC checklist compliance of the respondents varied between $25 \%$ and $63 \%$ averaging to $46.8 \%$ (Table I).

\section{Behavioural Assessment}

Utilizing the Likert's scale to assess the perceptions, attitudes and mindset of the 24 respondents, the most common barriers contributing to decreased compliance identified were confusion in ownership

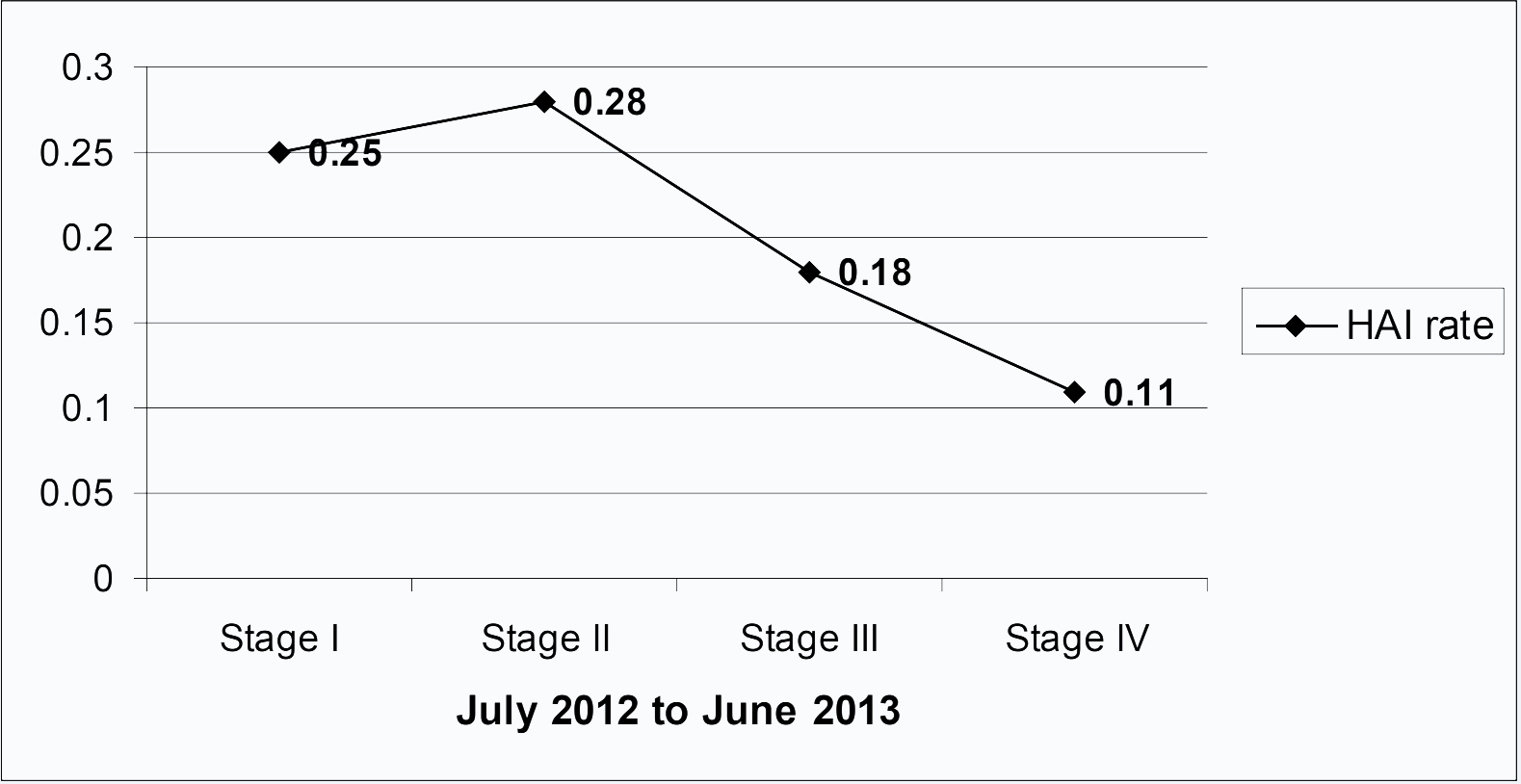

Figure 2. Trends of HAI rate from July 2012 to June 2013

Stage I- July-September 2012

Stage II- October-December 2012
Stage III- January-March 2013

Stage IV-April-June 2013 
Table I. Average Percentage Compliance (Hand Hygiene \& Infection Control Checklist) for Pre- and Post-training Periods

\begin{tabular}{|c|c|c|c|c|}
\hline \multirow{2}{*}{ S.No. } & \multicolumn{2}{|c|}{ Hand Hygiene Compliance } & \multicolumn{2}{|c|}{ Infection Control Checklist Compliance } \\
\hline & Pre-Training (\%) & Post-Training (\%) & Pre-Training (\%) & Post-Training* (\%) \\
\hline 1 & 35 & 55 & 25 & 30 \\
\hline 2 & 41 & 63 & 60 & 51 \\
\hline 3 & 38 & 78 & 55 & 65 \\
\hline 4 & 39 & 67 & 42 & 61 \\
\hline 5 & 31 & 58 & 35 & 54 \\
\hline 6 & 37 & 55 & 57 & 51 \\
\hline 7 & 36 & 51 & 38 & 43 \\
\hline 8 & 33 & 49 & 63 & 47 \\
\hline 9 & 32 & 57 & 48 & 52 \\
\hline 10 & 30 & 59 & 45 & 61 \\
\hline 11 & 32 & 64 & 47 & 55 \\
\hline 12 & 38 & 61 & 39 & 40 \\
\hline 13 & 47 & 68 & 61 & 63 \\
\hline 14 & 43 & 61 & 53 & 55 \\
\hline 15 & 55 & 55 & 57 & 56 \\
\hline 16 & 52 & 53 & 48 & 53 \\
\hline 17 & 44 & 69 & 61 & 52 \\
\hline 18 & 45 & 75 & 45 & 63 \\
\hline 19 & 42 & 71 & 47 & 51 \\
\hline 20 & 47 & 59 & 37 & 41 \\
\hline 21 & 39 & 61 & 33 & 52 \\
\hline 22 & 41 & 53 & 53 & 54 \\
\hline 23 & 35 & 65 & 35 & 41 \\
\hline 24 & 47 & 68 & 39 & 60 \\
\hline Mean & 40.0 & 61.5 & 46.8 & 52.1 \\
\hline
\end{tabular}

* Shaded boxes indicate the nurses who did not improve significantly after training 
and accountability in prevention of $\mathrm{HAI}$, lack of effective role models, perceived importance of IPC and the will to allot time to it, lack of repeated training and inadequate organizational encouragement and support. The training imparted to these nurses was focused to improve upon these behavioural stumbling blocks. This was done by targeting their mindsets and preconceived notions about taking up a responsibility as a frontline healthcare provider in preventing the patient from getting infected through a probable breach in IPC practices rather than emphasizing on teaching the set policies and protocols which they already knew.

\section{IPC practice measurement-Post training}

The hand hygiene compliance percentage of the nurses improved to $61.4 \%$ in this period (range- $49 \%$ to $78 \%$ ). This was a significant increase of $53.5 \%$ (from $40 \%$ in pre-training to $61.4 \%$ in the post training period, $\mathrm{p}<0.001$ ). The mean IC checklist compliance percentage post-training increased to $52 \%$ (range 30\% to $65 \%$ ). There was an $11.1 \%$ increase (from $46.8 \%$ in pre-training to $52.0 \%$ in post training, $\mathrm{p}$-0.015) in IC checklist compliance in the post training period which was significant. However, when the individual compliance results were assessed, it was found that only $79 \%$ (19 out of 24) of nurses have shown significant improvement in all aspects of competency in IPC. The remaining 21\% (5 out of 24) improved in hand hygiene but not in the IC checklist compliance.

\section{Outcome measurement}

The analysis of the HAI data for the given study period (July 2012-June 2013) was done in all the four stages (I-IV). A decline (Fig 2) was observed in HAI rates from stage III (behavioural training period) and continued thereafter. The HAI rates of first two quarters (prebehavioural training, mean-0.27) were compared to last two quarters (post-behavioural training, mean0.14 ) and the difference was found significant ( $p$ 0.010 ) with an overall reduction of $60.7 \%$.

\section{Discussion}

Infection prevention is an old science but a new art. Among the cornerstones of IPC, hand hygiene is the most important. The introduction of many initiatives and formulation of effective guidelines and standardized protocols have been developed in this field. Although knowledge and belief about the importance of infection control practices is high in some areas for preventing the HAls, there is much room for improvement in the actual practices. This requires effective methods to change the behaviour of health care workers (HCWs) towards IPC practices to reduce HAls and improve patients' safety, an area which is currently understudied. Among the HCWs, since nurses play a frontline role in prevention of infection, they become an important population to focus the study on. ${ }^{10}$

Our survey of the knowledge and awareness assessment reported that there exists incongruence between the knowledge about the IPC protocols and their actual practice in the nursing staff. This survey showed that all the selected nurses (24) were aware of the hand hygiene and IPC protocols. The level of awareness of these selected nurses was in the range of $50 \%$ to $95 \%$. However, the practice measurement reported that mean compliance to hand hygiene and IC checklist among nurses was only $40 \%$ and $46.8 \%$ respectively. Similar results have been reported be HICPAC in the past. ${ }^{11}$ Several other studies have reported low levels of compliance in spite of standardized protocols and guidelines. ${ }^{12-14}$ This showed that although the participants had adequate knowledge and awareness of the guidelines, protocols and their importance in reduction of HAls, the reported implementation of the same was low. The reason for low compliance to IPC practices in healthcare settings are related to psychological barriers, pre-conceived notions, cultural influences and ineffective time and resource management. ${ }^{15-18}$

In this study, we made an attempt to assess the psychological barriers among the nurses which may be responsible for the incongruence between and practice towards IPC by utilizing the behavioural assessment scale (BAS). The five common stumbling blocks identified by this were confusion of ownership and accountability, lack of effective role models, perceived importance of IPC and the will to allot time to it, lack of repeated training and inadequate organizational encouragement and support. Various studies carried out in this regard have reported inadequate facilities, ${ }^{19}$ lack of effective communication or knowledge, ${ }^{15,20}$ and time $^{20}$ as perceived barriers to optimal hand hygiene practices. The five barriers identified in this study were 
given emphasis and training imparted to the staff as an effort to remove them. A post behavioural training assessment of the IPC practices followed.

The post-training practice measurement reported a significant increase in the hand hygiene $(53.5 \%)$ and IPC practices (11.1\%) of the nurses. Several studies have reported similar results and it indicates that to bring a change in an individual's behaviour and practice one needs to target his mindset and perceptions. ${ }^{21,22}$ Several such behavioural interventions grounded on psychological frameworks were implemented in community settings and have proved successful. ${ }^{1}$

Another important finding reported in the study was that the overall improvement of the nurses postbehavioural training was significant but did not approach $100 \%$ as there were $79 \%$ (19 out of 24 ) of the nurses who improved overall and rest $21 \%$ (5 out of 24) showed improvement in hand hygiene but not in IC checklist compliance. This indicated that though as a group, they showed an increase in compliance, it was not $100 \%$ and 5 out of 24 respondents had not favourably responded to group behavioural therapy. Therefore, sometimes, individual perceptions and mindsets have to be worked upon in a one to one interaction.

The outcome measurement of the study reported that the effect of the behavioural intervention was positive in bringing about a decrease in HAI rates by $60.7 \%$ in the post-training period. This could be attributed to the significant increase in hand hygiene compliance $(53.3 \%)$ and IC checklist compliance $(11.1 \%)$ in this period.

The limitations of this study were that it was confined to the ICU and was carried out for a brief period of one year. Also, there could be more depth in the behavioural assessment to uncover other contributing factors to non-compliance. Furthermore, through this attempt we studied the first layer of the obvious behavioural attitudes of the healthcare practitioners (nurses). We intend to take this study forward in detail.

In conclusion, the present study highlights that understanding human behaviour leading to low compliance to IPC practices and imparting training to overcome these stumbling blocks can be a powerful, cost effective and rational approach to reduce HAls in low resource settings which eventually creates a culture of safety within the organization.

\section{Acknowledgement}

We owe our great thanks to Artemis Health Institute for extending their kind support and encouraging us in pursuing this study. The only source of funding for the present study was Artemis Health Institute. No external grants or funds have been obtained for the study.

\section{References}

1. Edwards R, Charani E, Sevdalis N, et al. Optimisation of infection prevention and control in acute health care by use of behaviour change: a systematic review. Lancet Infect Dis 2012; 12(4): 318-329. http://dx.doi.org/10.1016/S14733099(11)70283-3

2. Kennedy AM, Elward AM, Fraser VJ. Survey of knowledge, beliefs, and practices of neonatal intensive care unit healthcare workers regarding nosocomial infections, central venous catheter care, and hand hygiene. Infect Control Hosp Epidemiol 2004; 25: 747-752. http://dx.doi.org/10.1086/502471

3. McGlynn EA, Asch SM, Adams J, et al. The quality of healthcare delivered to adults in the United States. N Engl I Med 2003; 348: 2635-2645. http://dx.doi.org/10.1056/NEJMsa022615

4. Ferlie E, Fitzgerald L, Wood M, et al. The nonspread of innovations: the mediating role of professionals. Acad Management / 2005; 48: 117-134. http://dx.doi.org/10.5465/ AMJ.2005.15993150

5. Pittet D. The Lowbury lecture: behaviour in infection control. J Hosp Infect 2004; 58: 1-13. http://dx.doi.org/10.1016/j. jhin.2004.06.002

6. Gammon J, Morgan-Samuel H, Gould D. A review of the evidence for suboptimal compliance of healthcare practitioners to standard/ universal infection control precautions. I Clin Nurs 2007; 17: 157-167.

7. Aboelela SW, Stone PW, Larson EL. Effectiveness of bundled behavioural interventions to control healthcare-associated infections: a systematic review of the literature. / Hosp Infect 2007; 66: 101-108. http://dx.doi.org/10.1016/j. jhin.2006.10.019

8. Michie S, Van Stralen MM, West R. The behaviour change wheel: a new method for characterising and designing behaviour change interventions. Implement Sci 2011; 6: 42. http://dx.doi.org/10.1186/1748-5908-6-42

9. WHO guidelines on hand hygiene in healthcare-a summary. 2009 available at http://whqlibdoc.who.int/ publications/2009/9789241597906_eng.pdfReference

10. Sessa A, Di Giuseppe G, Albano L, et al. An investigation of nurses' knowledge, attitudes, and practices regarding disinfection procedures in Italy. BMC Infectious Diseases 2011, 11: 148. http://dx.doi.org/10.1186/1471-2334-11-148

11. Boyce JM, Pittet D; Healthcare Infection Control Practices Advisory Committee; HICPAC/SHEA/APIC/IDSA Hand Hygiene Task Force. Guideline for hand hygiene in healthcare settings: recommendations of the Healthcare Infection Control Practices Advisory Committee and the HICPAC/SHEA/ APIC/IDSA Hand Hygiene Task Force. Society for Healthcare Epidemiology of America/Association for Professionals in Infection Control/ Infectious Diseases Society of America. MMWR Recomm Rep 2002; 51: 1-45. 
12. Chan R, Molassiotis A, Chan E, et al. Nurses' knowledge of and compliance with universal precautions in an acute care hospital. Int / Nurs Stud 2002, 39: 157-163. http://dx.doi. org/10.1016/S0020-7489(01)00021-9

13. Nobile CGA, Montuori P, Diaco E, Villari P. Healthcare personnel and hand decontamination in intensive care units: knowledge, attitudes, and behaviour in Italy. I Hosp Infect 2002, 51: 226-232. http://dx.doi.org/10.1053/jhin.2002.1248

14. Chan MF, Ho A, Day MC. Investigating the knowledge, attitudes and practice patterns of operating room staff towards standard and transmission-based precautions: results of a cluster analysis. I Clin Nurs 2008, 17: 1051-1062. http:// dx.doi.org/10.1111/j.1365-2702.2007.01998.x

15. Sproat LJ, Inglis TJ. A multicentre survey of hand hygiene practice in intensive care units. J Hosp Infect 1994; 26: 137 148. http://dx.doi.org/10.1016/0195-6701(94)90057-4

16. Angelillo IF, Mazziotta A, Nicotera G. Nurses and hospital infection control: knowledge, attitudes and behaviour of Italian operating theatre staff. J Hosp Infect 1999; 42: 105112. http://dx.doi.org/10.1053/jhin.1998.0571
17. Houang ET, Hurley R. Anonymous questionnaire survey on the knowledge and practices of hospital staff in infection control. J Hosp Infect 1997; 35: 301-306. http://dx.doi.org/10.1016/ S0195-6701(97)90224-3

18. Gould D, Ream E. Nurses' views of infection control: an interview study. J Adv Nurs 1994; 19: 1121-1131. http:// dx.doi.org/10.1111/j.1365-2648.1994.tb01196.x

19. Scott G. Prevention and control of infections in intensive care. Intensive Care Med 2000; 26(suppl 1): S22-S25. http://dx.doi. org/10.1007/s001340051114

20. Pittet D. Compliance with hand disinfection and its impact on hospital acquired infections. J Hosp Infect 2001; 48(suppl A): S40-S46. http://dx.doi.org/10.1016/S0195-6701(01)90012-X

21. Kretzer EK, Larson EL. Behavioural interventions to improve infection control practices. Am J Infect Control 1998; 26: 245 53. http://dx.doi.org/10.1016/S0196-6553(98)80008-4

22. Bridle C, Riesma RP, Pattenden J, et al. Systematic review of the effectiveness of health behaviour interventions based on the transtheoretical model. Psychol Health 2005; 20: 283 301. http://dx.doi.org/10.1080/08870440512331333997 